\title{
AVALIAÇÃO DA SUSTENTABILIDADE CORPORATIVA E DA CULTURA ORGANIZACIONAL - SURVEY NO SETOR BRASILEIRO DE COSMÉTICOS
}

André Luiz Romano

andreromano1973@hotmail

Universidade Metodista de Piracicaba - UNIMEP- Piracicaba - SP - Brasil

Isabela Tatiana Teixeira

isabelatteixeira@hotmail.com

Universidade Federal de São Carlos - São Carlos - SP - Brasil

Alceu Gomes Alves Filho

alceu@ufscar.br ahelleno@unimep.br

Universidade Federal de São Carlos - São Carlos - SP - Brasil

\section{André Luís Helleno}

ahelleno@unimep.br

Universidade Metodista de Piracicaba - UNIMEP- Piracicaba - SP - Brasil

\section{Resumo}

Os benefícios oriundos das práticas de sustentabilidade são aqueles ligados a redução de custos, ampliação do volume de vendas, melhoria do clima organizacional, e o aumento das parcerias de ordem estratégicas. Sabe-se que esses benefícios podem relacionar-se a determinados tipos de cultura, que resumidamente pode ser definido, como o jeito no qual as coisas são realizadas em determinado ambiente, que vai desde uma casa, até um país, passando pelos diversos agentes envolvidos nas relações. Para se tratar da questão nas empresas, existe a chamada cultura organizacional. Aqui são apresentados os resultados da avaliação da cultura organizacional no setor de cosmético brasileiro, por meio da análise de correspondência. As análises mostram a existência de quatro grupos com associação entre posicionamentos. Verificou-se a associação entre empresas que consideram na maioria das vezes, os principais valores como liberdade e valorização das conquistas com empresas que tiveram benefícios muito grandes das práticas de sustentabilidade. Empresas com dificuldade de planejamento, estruturação, organização e capacidade analítica são empresas que tiveram apenas uma moderada melhoria no clima organizacional. Empresas sem a divisão de gênero e os funcionários podem falar diretamente com seus diretores são empresas que conseguiram uma ampliação muito grande do volume de vendas e expansão de mercado com a adoção dos programas de sustentabilidade. Nesse estudo, foi possível identificar importantes elementos da percepção gerencial sobre os benefícios oriundos de práticas de sustentabilidade no setor de cosmético brasileiro, não esgotando as possibilidades de análise, que podem ser aprofundadas e comparadas com os resultados de outras abordagens, como sugestão para futuras pesquisas no setor.

Palavras-chave: Cultura Organizacional. Sustentabilidade Corporativa. Setor de Cosmético.

\section{Abstract}

The benefits arising from the sustainable practices are those linked to cost savings, increased sales volume, improved organizational climate, and increasing strategic partnerships order. It is known that these benefits can be related to certain types of culture, which can be briefly defined 


\section{André Luiz Romano, Isabela Tatiana Teixeira, Alceu Gomes Alves Filho \\ André Luís Helleno}

as the way in which things are done in a particular environment, ranging from a house, even a country, through the various actors involved in relations. To address the issue in companies, there is the so-called organizational culture. The results of the assessment of organizational culture are presented here in the Brazilian cosmetic industry, through the correspondence analysis. The analyzes show that there are four groups of association between positions. It was the association between firms considering most of the time, the main values as freedom and appreciation of achievements with companies that have very large benefits of sustainability practices. Companies with limited planning, structuring, organization and analytical skills are companies that have only a moderate improvement in the organizational climate. Companies without a gender division and staff can speak directly to its directors are companies that have achieved a very large expansion of sales volume and market expansion with the adoption of sustainability programs. In this study, we identify important elements of managerial perception of the benefits arising from sustainability practices in the Brazilian cosmetic industry, not exhausting the possibilities of analysis, which can be studied and compared with the results of other approaches, such as suggestions for future research in the sector.

Keywords: Organizational culture.Corporate sustainability. Cosmetic industry.

\section{Introdução}

A incorporação de práticas de responsabilidade tem caracterizado o comportamento de empresas mais integradas e preocupadas com o desempenho organizacional. Estas práticas, quando apoiadas nas três dimensões da sustentabilidade corporativa (econômica, ambiental e social) podem levar a benefícios tangíveis e intangíveis para a organização. Contudo, surge na literatura uma discussão que inclui a cultura organizacional como dimensão da sustentabilidade no ambiente empresarial. Nessa pesquisa, se buscou o estudo da cultura organizacional e sua importância para o sucesso das práticas de sustentabilidade corporativa.

Conforme definido por Chiavenato (2010) a cultura organizacional é um conjunto de hábitos e crenças estabelecidos por normas, valores, atitudes e expectativas, divididos pelos membros de uma organização; refere-se a um grupo de significados compartilhados por todos os membros e que distingue uma organização das demais. A ideia de cultura organizacional surgiu entre as décadas de 1970 e 1980 (HOFSTEDE, 1981), (OUCHI; PRICE, 1993), (SCHWARTZ; DAVIS, 1981), (SCHEIN, 1992) como um dos mais controversos temas em gestão (CRANE, 1995). Desde o início houve uma ampla gama de interpretações que resultou numa falta de consenso: entre as definições de cultura incluem-se noções de comportamento, normas e rituais proposto por Trice e Beyer (1984), valores, ideologias e crenças sugeridos por Schwartz e Davis (1981) e formas compartilhadas de significado, como definido por Smircich (1983). Schein (1992) define pressupostos básicos, aqueles que constituem o núcleo de uma cultura de determinado grupo. Estes pressupostos ou paradigmas são as maneiras de sentir e perceber o ambiente no entorno de um determinado grupo. Schein (1992) afirma que a cultura pode ser separada em três níveis, que correspondem ao grau de visibilidade dos fenômenos organizacionais: i) artefatos; ii) valores; e iii) pressupostos básicos. Schein (2004) aponta esse terceiro nível, como o mais importante para a efetividade de uma mudança organizacional, envolvendo pressupostos básicos de um grupo, que serão tratados na revisão bibliográfica com maior detalhamento. Fleury e Fleury (1997) propõem a separação da cultura organizacional em três níveis: i) produtos e comportamentos visíveis; ii) histórias, mitos e heróis; e iii) valores. Segundo os autores, esses níveis apresentam uma relação direta com a proposta de Schein, existe uma contribuição importante deles, que é considerar a dimensão política no modelo. Segundo o instituto Ethos (2012), a RSE é uma forma de gestão baseada na relação ética e transparente da empresa e seus stakeholders, pelo estabelecimento de metas que levem ao desenvolvimento 
sustentável, preservando recursos ambientais e culturais, respeitando a diversidade e promovendo a diminuição das desigualdades sociais. Foi assumido nessa pesquisa que os benefícios em sustentabilidade podem ser percebidos de quatro maneiras pelas empresas: redução de custos a partir da adoção da sustentabilidade; ampliação das vendas, expansão de mercado com a sustentabilidade; melhoria no clima organizacional após a adoção da Sustentabilidade; e aumento de preferências do consumidor e produtividade. Com relação a avaliação da cultura organizacional, baseou-se no modelo de Hofstede (1980) e Alcântara et al. (2010) que descrevem cinco dimensões para avaliação da cultura organizacional, sendo eles: i) a hierarquia ou a distância do poder; ii) nível de individualismo; iii) flexibilidade ou nível de machismo da organização; iv) o grau de ameaça que o ambiente impõe a seus indivíduos; e v) a capacidade de inovação da organização determinada cultura tem.

Como objetivo principal desse artigo está a apresentação dos resultados obtidos por meio de um survey aplicado no setor de cosmético brasileiro. 0 intuito desse foi, por meio de uma análise estatística de correspondência, verificar quais as relações existentes entre a cultura organizacional e a sustentabilidade corporativa, determinando o grau de associação existente entre empresas com características homogêneas.

0 artigo está estruturado na seção 1 com a apresentação da pesquisa; na seção 2 estão os componentes dos benefícios das práticas de sustentabilidade, e seção 3, dando continuidade à revisão bibliográfica, há uma discussão acerca da cultura organizacional. A seção 4 descreve o setor de cosméticos, o método da pesquisa e os principais resultados do survey realizado. 0 artigo encerra-se com uma discussão sobre os resultados e as considerações finais do uso da análise de correspondência.

\section{Os Benefícios oriundos das práticas de sustentabilidade}

Kaptein e Wempe (2002) afirmam que a sustentabilidade corporativa pode contribuir para o desenvolvimento sustentável e que essa contribuição demandará um diálogo entre as visões de que, embora as empresas possam fazer mais do que apenas atender à requisitos atualmente vigentes, não são diretamente responsáveis por todos os impactos sociais e ambientais. Segundo Kaptein e Wempe (2002), a sustentabilidade corporativa, diferentemente da RSE, deve ir além da transparência e inclusão de stakeholders, comunicando ações sustentáveis, priorizando a criação de valor, práticas efetivas de gestão ambiental, sistemas de produção benéficos à natureza e uma eficiente gestão do capital humano.

A adoção de práticas de gestão dos recursos humanos tem sido inclusive vista como uma forma de vantagem competitiva do ponto de vista estratégico, conforme apontado por Jackson et al (2011). Os autores citam ainda que é necessário o entendimento da dinâmica das estruturas das grandes empresas e de como essas tem estabelecido padrões de gestão de seus recursos humanos, que afetarão num mundo globalizados todas as médias e pequenas empresas. Jabbour, Oliveira e Castro (2011) afirmam que a difusão da cultura organizacional podem encontrar resistência nas estruturas empresariais departamentalizadas, que podem fragmentar a cultura dessas empresas, gerando resistência a mudanças.

Sendo que a receptividade da organização, será elemento determinante de sucesso das mudanças.

Como em qualquer conceito ainda em construção, nota-se que a sustentabilidade corporativa não apresenta uma única e consensual definição, o que gera uma visão crítica na forma como se tem conduzido o conceito no ambiente empresarial. Mesmo entre autores que entendem os benefícios da sustentabilidade para a empresa e seu entorno, há discussões acerca da 


\section{André Luiz Romano, Isabela Tatiana Teixeira, Alceu Gomes Alves Filho \\ André Luís Helleno}

abrangência do conceito. É possível identificar grupos distintos de modelos, que consideram alguns aspectos em detrimento de outros. As relações entre a RSE e a sustentabilidade corporativa são representadas por meio da figura 1. A sustentabilidade corporativa trata-se de uma finalidade que deve ter em seus meios as ações de RSE.

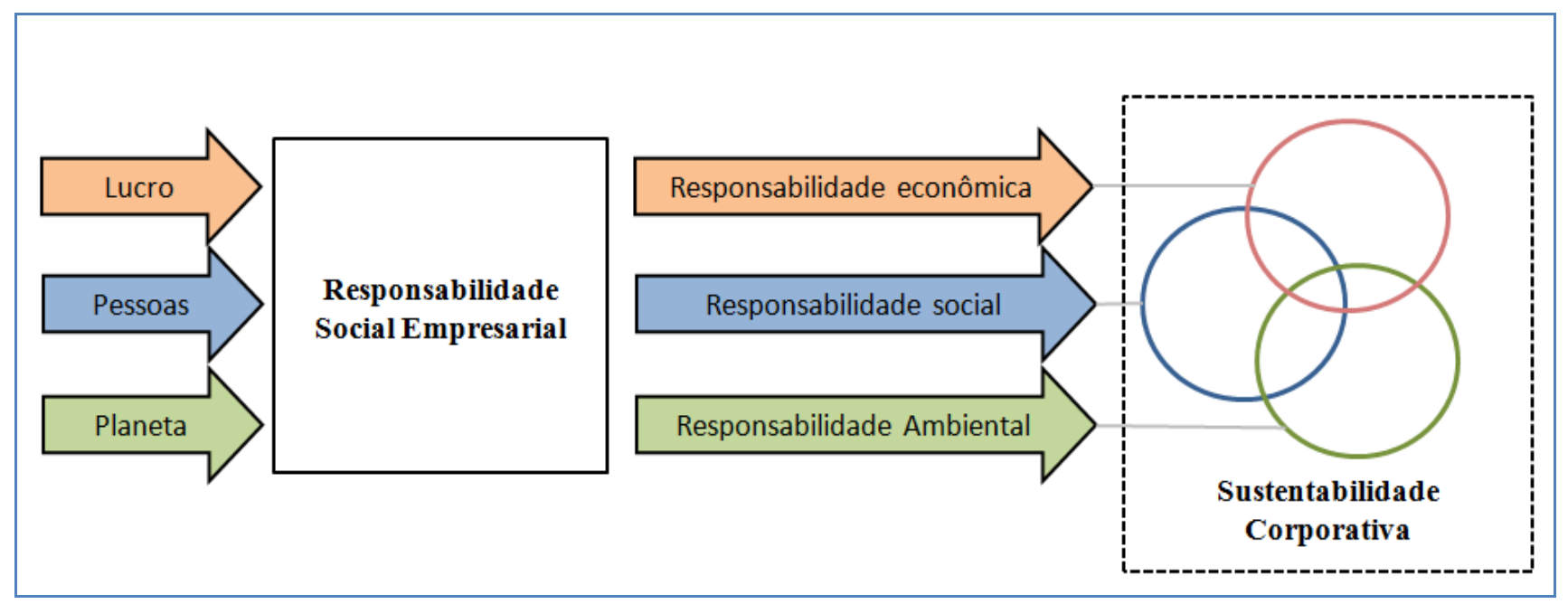

Figura 1 - Relação entre sustentabilidade corporativa e RSE

Fonte: Baseado em Kaptein e Wempe (2002); Savitz e Weber (2007).

De acordo com Dyllick e Hockerts (2002); Wright, Nyberg (2012); Dubois, e Dubois (2012); Chiappetta Jabbour, Almada Santos, e Fonseca (2013); Zellweger, Nason, Nordqvist (2013) a sustentabilidade é plenamente aceita, pois corresponde a um ideal de mundo mais evoluído socialmente, com maior justiça, que preserva o meio ambiente e culturas para as gerações vindouras. Entretanto, o objetivo de sustentabilidade não é tarefa simples, pois requer da sociedade abrir mão de parte significativa do conforto, riqueza e desenvolvimento. Existe a necessidade de mudança do foco intensivo em consumo, colocando as organizações diretamente no debate sobre como adotar a sustentabilidade, considerando que isso estará associado a um cenário de minimização do consumo e, consequentemente, da produção.

Azapagic (2003), Humphreys (2014), Srivastava, Franklin, e Martinette (2014) sugerem que a legislação deve ter papel decisivo no estabelecimento e promoção do desenvolvimento sustentável. A globalização e a complexidade que o mundo passou a ter com esse fato acentuou a importância da sustentabilidade, pois novas demandas fizeram emergir empresas com ferramentas de comprovação do grau de transparência de suas operações. As empresas ganham importância a cada dia, por vezes ocupando o papel social do Estado, e isto certamente se explica ao se verificar que, entre as 100 maiores economias do mundo, mais da metade são corporações.

$\mathrm{Na}$ pesquisa dos autores que tratam o tema sustentabilidade corporativa, identificam-se diferentes modelos e propostas que mostram a relevância da sustentabilidade no meio empresarial. Shrivastava (1995), Bansal e Roth (2000), Elkington (1997), (2001), Epstein e Roy (2001), Dyllick e Hockerts (2002), Hart e Milstein (2004), Bansal (2005), Salzmann, IonescuSomers e Steger (2005), Isaksson (2006), Wagner (2010), Berns et al (2012), Mendiola, Beltran, e Tirados (2013) e Romano (2010) e (2014) apontam para uma série de fatores que conduzirão as empresas de forma espontânea a responderem as demandas de sustentabilidade e a deterem uma primazia em seus modelos de negócio. Conforme apontado na figura 2, a relação entre as variáveis de sustentabilidade corporativa está nas diferentes dimensões (ELKINGTON; 1997). 


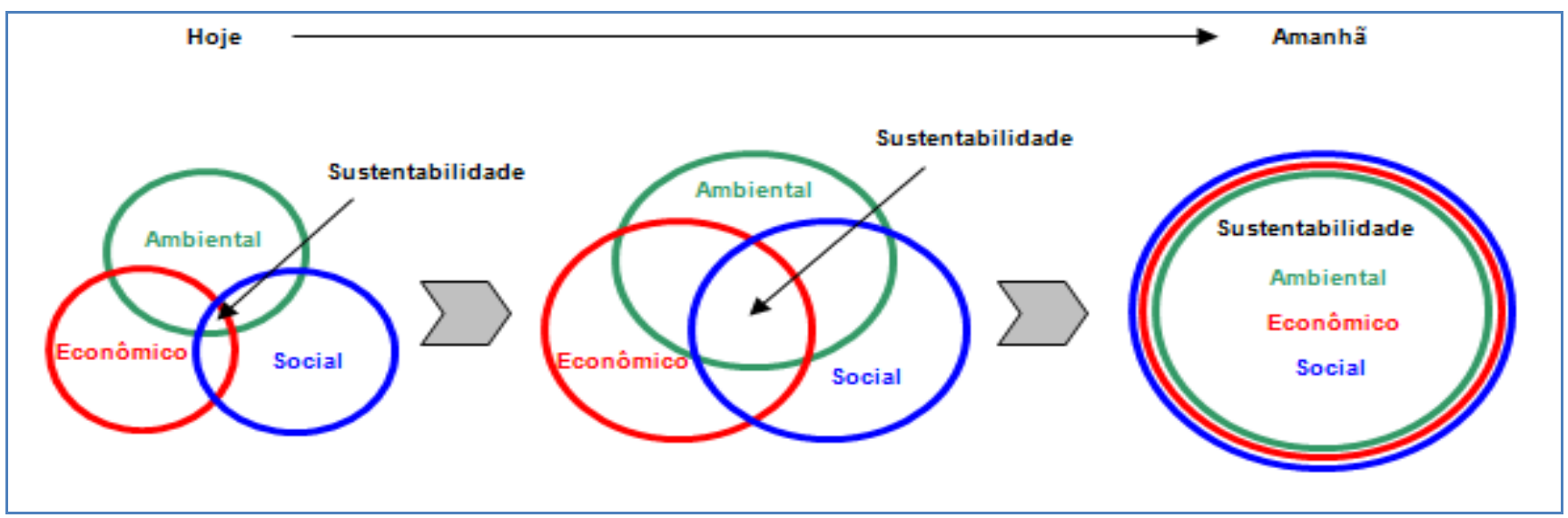

Figura 2 - Alinhamento das dimensões da sustentabilidade corporativa Fonte: Adaptado de Elkington (2001).

As empresas que adotarem programas de RSE terão que passar por uma transição de um modelo que trate as dimensões de forma quase isoladas, com pouca interseção, para um modelo que considere dimensões econômicas, sociais e ambientais. Essa transição ganha ainda maior complexidade, pois envolve toda a cadeia de negócios das empresas, o que afeta as relações com fornecedores, parceiros e clientes. Savitz e Weber (2007), Weinstein, Turner, e Ibanez (2013), Kiron et al (2014) descrevem que a sustentabilidade corporativa ou Triple Bottom Line (TBL) captura a essência da sustentabilidade por mensurar os impactos das atividades de uma empresa em seu entorno. Segundo os autores, uma empresa que tiver TBL positivo terá um aumento no valor da organização.

A sustentabilidade corporativa é um processo dinâmico, que leva às entidades empresariais a legitimação de objetivos econômicos, desde que compartilhem os ganhos com seus stakeholders. Ao longo do amadurecimento teórico do tema, outros autores passam a considerar também dimensões adicionais àquelas propostas inicialmente e que, numa linguagem simplificada, seriam desdobramentos da visão proposta por Elkington. Sachs (2000) apresenta, conforme representado na figura 3 , uma contribuição para a abordagem da sustentabilidade.

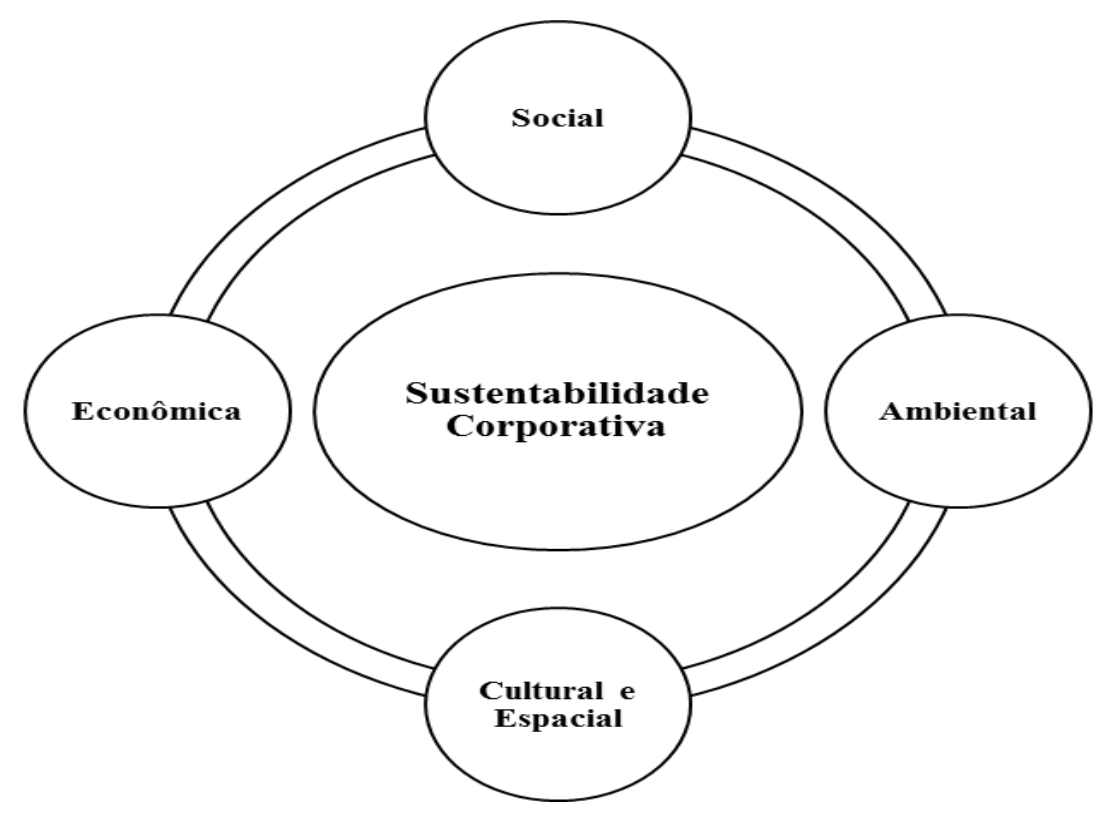

Figura 3 - As dimensões da sustentabilidade corporativa

Fonte: Adaptado de Sachs (2000). 


\section{André Luiz Romano, Isabela Tatiana Teixeira, Alceu Gomes Alves Filho \\ André Luís Helleno}

Segundo Sachs (2000), a Sustentabilidade Corporativa deve considerar as seguintes dimensões: i) econômica - possibilita uma alocação e gestão mais eficiente dos recursos e um fluxo regular de investimentos públicos e privados; ii) social - consolida um processo de desenvolvimento baseado num tipo de crescimento orientado por uma visão do que é uma "boa" sociedade; iii) ambiental - minimiza o dano aos ecossistemas, reduzindo impactos e o consumo de recursos não renováveis, considera práticas de reciclagem e conservação, investindo em tecnologias mais limpas; com regras de proteção ambiental; iv) cultural - respeito às especificidades de cada ecossistema, de cada cultura e de cada local e v) espacial - volta-se para uma configuração ruralurbana mais equilibrada e uma melhor distribuição territorial das atividades humanas.

A proposta de Sachs (2000) difere da construção de Elkington, pois apresenta uma abordagem direcionada para os recursos, apontando uma fragilidade do atual modelo, pois, ao se aumentar o volume de produção a pretexto de gerar maior resultado econômico, ocorrerá também um aumento da destruição dos recursos naturais, que são fundamentais para as futuras gerações.

Sachs afirma que a dimensão cultural apresenta particularidades regionais, pois, por exemplo, uma área de influência da agropecuária está submetida a uma realidade econômica e sociocultural distinta de regiões de base industrial. Outra dimensão que, para Sachs, apresenta diferença é a espacial e política, relacionada com a autoestima e cidadania, geralmente devido a um estado controlador dos recursos, a pretexto de decisões políticas.

A dimensão cultural é citada também por Veiga (2007), Harris e Crane (2002), e Baumgartner (2014) que destacam a importância dessa dimensão para a estruturação da sustentabilidade. Segundo Veiga (2007), existe uma ampla gama de conceitos sob os quais a sustentabilidade está estruturada, diretamente relacionada e dependente de aspectos históricos, sociais, econômicos e culturais, impossibilitando que se trate a sustentabilidade como algo linear ou exato.

A importância do aspecto cultural pode ser constatada por Harris e Crane (2002), ao afirmarem que, para atingir a sustentabilidade corporativa, as empresas precisarão contar com soluções técnicas, mas fundamentalmente com novos valores, crenças e comportamentos. Entende-se com isso que o sucesso da sustentabilidade corporativa tem relação direta com o grau de mudança da cultura organizacional e essas mudanças não podem ser apenas superficiais ou aparentes. Uma das lacunas identificadas nessa pesquisa está no entendimento da percepção que os gestores têm acerca da sustentabilidade e quais são os fatores que facilitam ou dificultam o sucesso da sustentabilidade no âmbito corporativo.

\section{A Cultura Organizacional}

A cultura organizacional se forma por intermédio da vivência e experiência do grupo em lidar com problemas, sejam eles dentro do processo de aprendizagem segundo o qual se resolvem problemas ou não. Hofstede (1998) aponta cultura como uma característica coletiva e não individual. Fleury e Fleury (1997) afirmam que a cultura se refere aos valores profundos de uma organização, desenvolvidos e assimilados pelo grupo ao longo da sua história, e é a forma como uma organização aprendeu a lidar com os problemas (CRANE, 1995), (LINNENLUECKE; RUSSELL; GRIFFITHS, 2009).

Schein (1992) propõe um modelo teórico de cultura organizacional composto por três níveis ou dimensões, conforme apresentado na figura 4. 


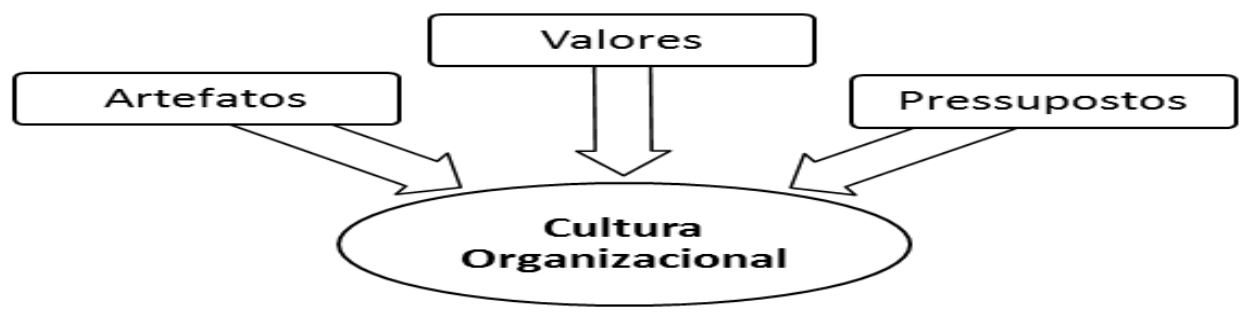

Figura 4 - Componentes da cultura organizacional Fonte: Baseado em Schein (2004).

Esses três níveis correspondem ao grau de visibilidade que os fenômenos organizacionais apresentam: i) artefatos - incluem os elementos tangíveis, identificáveis de maneira simples numa organização (arquitetura, mobília, código de vestimenta). Trata-se dos elementos visíveis de uma cultura e podem ser reconhecidos por pessoas fora dessa cultura; ii) valores - são os valores declarados por uma organização, como as regras de comportamento. Corresponde à forma como os indivíduos apresentam a organização, tanto para si mesmos, quanto para os outros; e iii) pressupostos básicos - é o aspecto com maior profundidade em cultura. São as ações tomadas de maneira normalmente inconsciente e correspondem à essência dessa cultura, constituindo a parte de maior dificuldade na sua identificação.

Uma organização deve zelar objetivamente pelo correto gerenciamento do terceiro nível. Segundo Schein (2004), é o mais importante para a efetividade de uma mudança organizacional, pois envolve pressupostos básicos de um grupo. Os artefatos são os aspectos visíveis, audíveis, manifestações dos pressupostos básicos, como padrões de comportamento, rituais, ambiente físico, histórias e mitos.

Podem ser, por exemplo, como os códigos de vestimenta identificados em algumas empresas, que representam a indicação de alguma cultura básica.

Os valores compartilhados compreendem outro componente da cultura organizacional, cujas razões expostas explicam por que as coisas são da forma que são, tais como normas internas, códigos de ética, formalização de valores da companhia e outros; Como exemplo, muitas empresas possuem uma a missão declaradamente, informando aos clientes e colaboradores o que a empresa espera, e vinculando elementos essenciais da cultura organizacional em questão.

Baumgartner (2014) apresenta uma abordagem que trata da influência que os programas de sustentabilidade sofrem dos artefatos, valores e pressupostos básicos, que são aspectos culturais de uma organização. Um programa de sustentabilidade afetará as relações de poder dentro da organização, e, sobretudo por se tratar de um conceito novo, é natural que existam resistências de pessoas ou departamentos, que se sentem como se estivessem perdendo seu poder (por exemplo, na condução de um programa de sustentabilidade, as pessoas ou setores que estiverem liderando o projeto terão um maior volume de recursos financeiros à sua disposição, além de maior visibilidade, o que certamente resultará em maior força para impor suas prioridades e objetivos, desencadeando as tais resistências de pessoas ou departamentos).

Um adequado gerenciamento deste processo de mudança identificará as resistências prováveis e terá que lidar com elas, embora não haja consenso por parte dos autores sobre a melhor forma de lidar com essas resistências. Linnenluecke e Griffiths (2010) apontam que a cultura organizacional é frequentemente tratada como a principal razão do fracasso da adoção de programas de mudança organizacional. 


\section{André Luiz Romano, Isabela Tatiana Teixeira, Alceu Gomes Alves Filho \\ André Luís Helleno}

As significativas trocas de sistemas e de métodos nas empresas devem ser precedidas pelas mudanças na cultura organizacional instalada. Ao não se trabalhar a cultura do meio organizacional, novas técnicas adotadas podem não ter o sucesso esperado. No momento que não se modificam os pressupostos básicos, as mudanças serão apenas superficiais e a organização estará desperdiçando tempo com ideais não se sustentarão. Determinados tipos de culturas influenciam decisivamente o sucesso de adoção de novos modelos de negócios. Uma empresa com cultura mais permeável terá maior chance de sucesso, pois terá uma maior disseminação e absorção do que uma cultura fechada e conservadora.

A cultura organizacional pode ser mensurada pelas dimensões identificadas por Hofstede (1980) e Alcântara et al. (2010) em pesquisas multiculturais. Silva, Medeiros e Enders (2011) apontam que a uma abordagem quantitativa possibilita a descrição da cultura de um ponto de vista do indivíduo externo à organização. Homburg e Pflesser (2000) demonstram que o uso de tipologias quantitativas possibilita uma maior compreensão do fenômeno, ao passo que Schein (1992) considera que podem apresentar um risco de reducionismo que pode prejudicar a compreensão da complexidade da cultura.

Hofstede (1980), que realizou comparações entre culturas nacionais e em empresas multinacionais em vários países, detalhando cinco dimensões culturais: distância hierárquica, individualismo, masculinidade, controle da incerteza e visão de curto e longo prazo. Essas dimensões são mensuradas e viram índices que servem para identificar a cultura predominante, conforme o quadro 1.

\begin{tabular}{|c|c|c|}
\hline : & $\begin{array}{l}\text { Características de Sistema } \\
\text { Flexível }\end{array}$ & $\begin{array}{l}\text { Características de Sistema } \\
\text { Rígido }\end{array}$ \\
\hline 䄈 & $\begin{array}{l}\text { Pequena distância hierárquica } \\
\text { - } \quad \text { Desigualdades devem ser mínimas; } \\
\text { - } \quad \text { Interdependência dos níveis de poder; } \\
\text { - Hierarquia é forma de desigualdade; } \\
\text { - A descentralização é comum; } \\
\text { - Diferença salarial mínima entre níveis; } \\
\text { - Subordinados esperam ser consultados; } \\
\text { - O chefe ideal é democrata competente; } \\
\text { - } \quad \text { Privilégios e “status” são mal vistos. }\end{array}$ & $\begin{array}{l}\text { Grande distância hierárquica } \\
\text { - } \quad \text { Desigualdades são desejáveis. } \\
\text { - } \quad \text { Dependência dos níveis de poder; } \\
\text { - } \quad \text { Hierarquia é desigualdade existencial; } \\
\text { - } \quad \text { A centralização é comum; } \\
\text { - } \quad \text { Diferença salarial elevada entre níveis; } \\
\text { - } \quad \text { Subordinados esperam ser orientados; } \\
\text { - } \quad \text { o chefe ideal é autocrata benevolente; } \\
\text { - } \quad \text { Os privilégios "status" são essenciais. }\end{array}$ \\
\hline$\overline{\mathrm{Z}}$ & $\begin{array}{l}\text { Sociedades coletivas } \\
\text { - } \quad \text { Cuidam entre si, lealdade e troca; } \\
\text { - } \quad \text { identidade é função do grupo social; } \\
\text { - Harmonia, evitando confrontos diretos; } \\
\text { - } \quad \text { Emprego em condições morais; } \\
\text { - } \quad \text { Decisões consideram grupos; } \\
\text { - } \text { Administrar relacionamentos; } \\
\text { - } \quad \text { A relação prevalece sobre a tarefa. }\end{array}$ & $\begin{array}{l}\text { Sociedades individualistas } \\
\text { - } \quad \text { Pessoa cresce, e cuida de si e dos seus; } \\
\text { - } \quad \text { A identidade baseada no individuo; } \\
\text { - } \quad \text { Dizer o que pensa é para os honestos; } \\
\text { - } \quad \text { Emprego em condições de vantagens; } \\
\text { - } \quad \text { Decisões de acordo com as regras; } \\
\text { - } \\
\text { - } \\
\text { - }\end{array}$ \\
\hline
\end{tabular}




\section{AVALIAÇÃO DA SUSTENTABILIDADE CORPORATIVA E DA CULTURA ORGANIZACIONAL - SURVEY NO SETOR BRASILEIRO DE COSMÉTICOS}

\begin{tabular}{|c|c|c|}
\hline & $\begin{array}{l}\text { Sociedades femininas } \\
\text { - Valores: atenção e cuidado pelos outros; } \\
\text { - Homens e mulheres podem ser ternos; } \\
\text { - Trabalhar para viver; } \\
\text { - Intuição e a qualidade de vida no } \\
\text { trabalho; } \\
\text { - Igualdade, solidariedade, qualidade de } \\
\text { vida; } \\
\text { - Conflitos resolvidos com negociação; } \\
\text { - Todos devem ser modestos; } \\
\text { - Importância de pessoas, relações } \\
\text { calorosas. }\end{array}$ & $\begin{array}{l}\text { Sociedades masculinas } \\
\text { - } \quad \text { Valores: sucesso e progresso material; } \\
\text { - } \quad \text { Mulheres são ternas e relacionam-se; } \\
\text { - } \quad \text { Viver para trabalhar; } \\
\text { - } \quad \text { Decisivos e auto afirmativos; } \\
\text { - } \quad \text { Equidade, competição e desempenho; } \\
\text { - } \quad \text { Conflitos resolvidos por confronto; } \\
\text { - } \quad \text { Autoconfiança, ambição e dureza; } \\
\text { - } \quad \text { A importância do dinheiro e bens. }\end{array}$ \\
\hline ত্ & $\begin{array}{l}\text { Baixo controle da incerteza } \\
\text { - } \quad \text { Pouco stress, bem-estar; } \\
\text { - } \quad \text { Emoção e agressão minimizadas; } \\
\text { - } \quad \text { Educação flexível ao que é sujo e tabu; } \\
\text { - } \quad \text { O que é diferente é curioso; } \\
\text { - } \quad \text { Menor número possível de regras; } \\
\text { - } \quad \text { Tempo é um marco de referência; } \\
\text { - } \quad \text { Trabalha-se muito quando necessário; } \\
\text { - } \quad \text { Precisão e pontualidade são aprendidas; } \\
\text { - } \quad \text { Tolerância e moderação prevalecem. }\end{array}$ & $\begin{array}{l}\text { Elevado controle da incerteza } \\
\text { - } \quad \text { Stress elevado, ansiedade; } \\
\text { - } \quad \text { Emoções e agressão podem ocorrer; } \\
\text { - } \quad \text { Normas estritas sobre tabu e sujo; } \\
\text { - } \quad \text { O que é diferente é perigoso; } \\
\text { - } \quad \text { Demanda regras, mesmo ineficazes; } \\
\text { - } \quad \text { Tempo é dinheiro. } \\
\text { - } \quad \text { Necessidade emocional de ocupação; } \\
\text { - } \quad \text { Precisão e pontualidade são naturais; } \\
\text { - } \quad \text { Resistência para inovação. }\end{array}$ \\
\hline$\sum$ & $\begin{array}{l}\text { Visão de Curto Prazo } \\
\text { - } \quad \text { Valores: liberdade, respeito aos direitos. } \\
\text { - } \quad \text { Devem produzir resultados rápidos. } \\
\text { - } \quad \text { Foco na geração dos lucros deste ano. } \\
\text { - } \quad \text { Gestores e funcionários são diferentes. } \\
\text { - } \quad \text { Recompensa: meritocracia, por } \\
\text { habilidades. } \\
\text { - } \quad \text { Lealdade atende demandas do negócio. } \\
\text { - } \quad \text { A preocupação em possuir a Verdade. } \\
\text { - } \quad \text { Pensamento analítico. }\end{array}$ & $\begin{array}{l}\text { Visão de Longo Prazo } \\
\text { - Valores: honestidade, autodisciplina, } \\
\text { responsabilidade. } \\
\text { - } \quad \text { O foco está em crescer, ganhar mercado. } \\
\text { - Importância dos lucros daqui a dez anos. } \\
\text { - Todos compartilham mesmas aspirações. } \\
\text { - Satisfação em corrigir injustiças. } \\
\text { - } \quad \text { A prioridade é dada ao bem comum. } \\
\text { - Desacordo não faz mal. } \\
\text { - } \quad \text { Pensamento sintético. }\end{array}$ \\
\hline
\end{tabular}

Quadro 1 - Organizações baseadas na flexibilidade ou no controle

Fonte: Adaptado de Hofstede (1980); Alcântara et al. (2010).

A hierarquia foi o primeiro elemento identificado, podendo ser também reconhecida como a distância do poder. Para se determinar a hierarquia são utilizados aspectos como, quanto os membros menos poderosos aceitam e esperam distribuição desigual de poder; qual o sistema de valores daqueles que têm menos poder. A segunda dimensão identificada foi o nível de individualismo, que aborda até que ponto as pessoas sentem que podem tomar conta de si 


\section{André Luiz Romano, Isabela Tatiana Teixeira, Alceu Gomes Alves Filho \\ André Luís Helleno}

próprias, das suas famílias ou das empresas.

A terceira dimensão é a flexibilidade da organização, que aborda o nível de machismo incutido na organização. Por exemplo, como a o poder está concentrado nas mãos dos homens, quão agressivo e competitivo é o seu comportamento e o quando negocia, como também se está mais interessado em resultados ou em justiça. Na quarta dimensão está o grau de ameaça que o ambiente impõe a seus indivíduos, podendo refletir um ambiente de desconforto, mediante a sensação de riscos, caos e situações não estruturadas. Adicionalmente, esses sentimentos são expressos por meio de estresse nervoso e uma necessidade de previsibilidade, como regras claras e escritas. A quinta dimensão relaciona-se à capacidade de inovação que determinada cultura tem. É difícil inovar em ambientes que pensem no curto prazo, pois apresentam valores orientados ao passado e o presente como respeito pela tradição e cumprimento de obrigações sociais. Empresas inovadoras que apresentam culturas de longo prazo têm valores orientados para o futuro, como economia (poupança) e persistência.

Da caracterização de cada perfil, espera-se que haja um tipo que possa ser favorável para o sucesso da sustentabilidade corporativa.

\section{Aplicação de uma averiguação no setor de cosmético brasileiro}

O segmento de cosmético faz parte do setor denominado Higiene Pessoal, Perfumaria e Cosméticos (HPPC). Segundo a ABIHPEC, esse setor apresentou um crescimento médio de 10,09\% entre 1996 e 2011, e passou de um faturamento líquido de imposto sobre vendas de MMR\$ 4,9 em 1996, para MMR\$ 29,4 em 2011. Para a ABIHPEC (2012) o crescimento desse setor se deve a fatores como a crescente participação da mulher no mercado de trabalho e a utilização de alta tecnologia.

O aumento da produtividade é responsável pelos preços do setor apresentar inflação menor do que os índices gerais da economia. Outro aspecto que contribui para o seu crescimento é o aumento da expectativa de vida, que implica na necessidade de conservar uma impressão de juventude. De acordo com a ABIHPEC (2012), há 1.659 empresas no setor de higiene pessoal, perfumaria e cosméticos; dessas, 330 são classificadas pela CosméticosBR (http://www.cosmeticosbr.com/index.asp) como produtoras e comercializadoras de produtos acabados.

O grupo de produtos acabados foi convidado a participar da presente pesquisa; o questionário foi encaminhado a 330 empresas, as quais retornaram 102 respostas. Os dados foram coletados por meio do questionário on-line do site www.surveymonkey.com.br aplicado entre Maio e Julho de 2013, e parte aplicada via telefone/e-mail pelo pesquisador. 0 objetivo do questionário foi verificar a percepção sobre cultura organizacional das empresas de cosméticos que atuam no Brasil. Foi utilizada uma escala crescente de concordância de cinco pontos.

\section{Métodos de Pesquisa}

A opção por um estudo no setor de cosméticos se deu em função da importância que a sustentabilidade vem recebendo nesse segmento. Trata-se de um setor no qual a imagem tem papel essencial no valor de um produto, sendo cada vez mais valorizada pelos consumidores. 0 intuito do artigo foi averiguar respostas representativas do setor por meio do uso de instrumental estatístico e processamento de dados numéricos, que possibilitassem a realização de inferências para o entendimento dos relacionamentos existentes entre as variáveis. 0 objetivo foi que os resultados representassem a realidade das empresas do setor de cosméticos, o que caracteriza a abordagem dessa etapa da pesquisa como quantitativa. 
Para a aplicação da abordagem quantitativa, optou-se pelo método de pesquisa survey, que é caracterizado pelo uso de variáveis bem definidas e generalização de resultados obtidos para o setor (MARTINS, 2010). 0 survey foi conduzido por meio de um questionário auto administrado, enviado por e-mail, em link, para que fosse respondido diretamente no site do repositório contratado.

O método escolhido para avaliação dos dados coletados foi a análise de correspondências múltiplas, técnica de interdependência que tem ganhado popularidade na redução dimensional e mapeamento perceptual.

\section{Analise de correspondências múltiplas}

A análise de correspondência foi utilizada para a averiguação dos dados; esta se trata de uma estatística do grupo da análise multivariada, um método de redução de dados que objetiva a obtenção de agrupamentos de dados similares entre si, com a finalidade de classificar conforme as relações naturais da amostra, formando grupos de objetos (Benefícios das Práticas de Sustentabilidade e Cultura Organizacional).

No quadro 2, são apresentadas as questões de cultura, identificadas com o código B (sendo composta pelos itens apresentados no quadro 1) e as questões de benefícios em sustentabilidade como sendo as questões do código D, com quatro questões sobre benefícios oriundos das práticas de sustentabilidade corporativa.

\begin{tabular}{|c|c|}
\hline Variável & uestão relacionada \\
\hline Bloco B & $\begin{array}{l}\text { E1 = QB1 => A influência de uma pessoa é baseada primeiramente em seu cargo. } \\
\text { E1 = QB2 => Funcionários não possuem liberdade para questionar as ordens superiores. } \\
\text { E1 = QB3 => Os funcionários não podem falar diretamente com seus diretores. } \\
\text { E1 = QB4 => As posições mais altas tem privilégios diferentes daqueles das mais baixas. } \\
\text { E2 = QB5 => Impera o individualismo e egoísmo nas relações profissionais. } \\
\text { E2 = QB6 => } 0 \text { indivíduo é mais valorizado que o grupo. Não existe trabalho em grupo. } \\
\text { E2 = QB7 => Os interesses individuais sobrepõe os coletivos. } \\
\text { E2 = QB8 => As relações de competição profissional são claramente percebidas. } \\
\text { E3 = QB9 => A maioria dos funcionários caracteriza-se por indelicadeza. } \\
\text { E3 = QB10 => Existem poucas ou nenhuma mulher em cargos gerenciais. } \\
\text { E3 = QB11 => A maioria dos funcionários esconde as suas emoções. } \\
\text { E3 = QB12 => Ocorre uma divisão clara dos papéis entre homens e mulheres. } \\
\text { E4 = QB13 => Quase nunca os funcionários se sentem nervosos. (perder emprego). } \\
\text { E4 = QB14 => 0s funcionários preferem tarefas bem estruturadas, com ordens claras. } \\
\text { E4 = QB15 => Grande parte do trabalho é bem planejada, levando a poucas surpresas. } \\
\text { E4 = QB16 => As reuniões frequentemente são planejadas com antecedência. } \\
\text { E5 = QB17 => Produzir resultados rápidos. O foco deste ano está na geração de lucros. } \\
\text { E5 = QB18 => Lealdades pessoais variam de acordo com as necessidades do negócio. } \\
\text { E5 = QB19 => Principais Valores são liberdade e a valorização das conquistas. } \\
\text { E5 = QB20 => 0 pensamento é na maioria das vezes analítico. }\end{array}$ \\
\hline
\end{tabular}




\section{André Luiz Romano, Isabela Tatiana Teixeira, Alceu Gomes Alves Filho \\ André Luís Helleno}

Bloco D

$$
\begin{aligned}
& \text { E17 = QD1 => Houve redução de custos a partir do Programa de sustentabilidade? } \\
& \text { E18 = QD2 => Houve ampliação ou expansão de mercado com a sustentabilidade? } \\
& \text { E19 = QD3 => Houve melhoria no clima organizacional com a sustentabilidade? } \\
& \text { E20 = QD4 => Aumentaram de parcerias (fornecedores/clientes) com a sustentabilidade? }
\end{aligned}
$$

Quadro 2 - Relação entre Variáveis e Questões

Fonte: Próprio autor.

Segundo Hair et al (2006) a análise de correspondência permite a melhor visualização, das associações existentes entre as variáveis como as de cultura organizacional com os Benefícios percebidos pelas empresas em termos de Sustentabilidade. Na figura 5 são apresentados, por meio dos códigos das questões inseridas no sistema $\mathrm{R}$, os resultados que constam do documento original da tese.

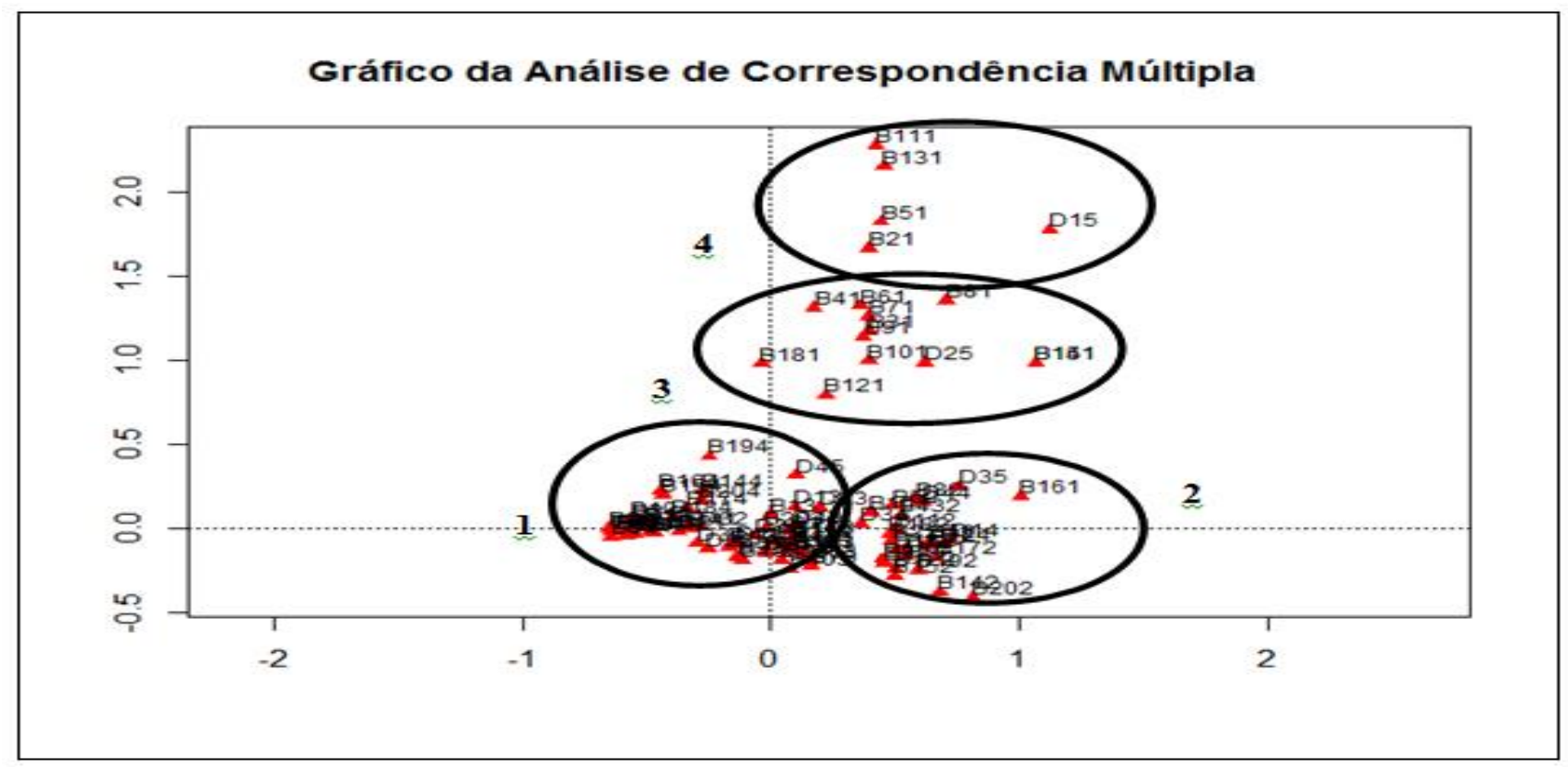

Figura 5 - Correspondência entre cultura e benefícios em sustentabilidade

Fonte: Elaborado pelo autor

O primeiro grupo (1) é formado pelas empresas que consideram na maioria das vezes, os principais valores como liberdade e valorização das conquistas; com a maioria dos funcionários, na maioria das vezes, preferindo tarefas bem estruturadas, com ordens claras; empresas que tiveram um aumento muito grande nas preferências do consumidor, competitividade e produtividade após a adoção de um programa de sustentabilidade; melhoria moderada no clima organizacional após a adoção do programa de Sustentabilidade e ampliação moderada do volume de vendas e expansão de mercado com a adoção do programa. Essas são as características que foram possíveis serem identificadas no gráfico. 0 grupo (2) é formado pelas empresas em que as reuniões nunca são planejadas com antecedência; o pensamento, na minoria das vezes é para a maioria dos respondentes é analítico; na minoria das vezes os funcionários preferem que as tarefas sejam bem estruturadas, com ordens claras; na minoria das vezes as relações de competição profissional são claramente percebidas e houve melhoria moderada no clima organizacional após a adoção do programa de Sustentabilidade. 0 grupo (3) é formado pelas empresas em que as lealdades pessoais nunca variam de acordo com as necessidades do negócio; nunca ocorre divisão dos papéis entre homens e mulheres; existem várias mulheres em cargos administrativos; grande parte do trabalho nunca é bem planejada; a maioria dos funcionários nunca se caracteriza por indelicadeza; os funcionários podem falar 
diretamente com seus diretores; os interesses individuais nunca sobrepõem os coletivos; as posições mais altas nunca têm privilégios diferentes daqueles das posições mais baixas; o indivíduo nunca é mais valorizado que o grupo; as relações de competição profissional não são claramente percebidas e houve uma ampliação muito grande do volume de vendas e expansão de mercado com a adoção do programa. Por fim, o quarto grupo (4) é caracterizado por funcionários que nunca escondem suas emoções; que se sentem tensos e nervosos; que nunca usam individualismo e egoísmo em suas relações profissionais; que possuem sempre liberdade para questionar as ordens de seus dirigentes e que tiveram redução muito grande de custos a partir da adoção do programa de sustentabilidade.

\section{Discussões e considerações finais}

A aplicação do Survey no setor de cosmético brasileiro permitiu o entendimento da percepção gerencial de 102 empresas do setor sobre o tema benefícios e cultura organizacional oriundos de práticas de sustentabilidade corporativa. Como resultado geral foi identificado uma associação entre empresas que consideram entre os principais valores a liberdade e valorização das conquistas, cujos funcionários preferem tarefas bem estruturadas e com ordens claras, com empresas que tiveram um aumento muito grande nas preferências do consumidor, na competitividade e na produtividade; dessa relação, apontou-se melhoria moderada no clima organizacional, ampliação moderada do volume de vendas e expansão de mercado com a adoção do programa de sustentabilidade.

Conclui-se também que as empresas com dificuldade de planejamento, estruturação, organização e capacidade analítica são aquelas que tiveram apenas uma moderada melhoria no clima organizacional. Já em empresas sem a divisão de gênero, nas quais não existe a variação da lealdade de acordo com as necessidades do negócio, nas quais os funcionários podem falar diretamente com seus diretores, o indivíduo nunca é mais valorizado que o grupo e as relações de competição profissional não são claramente percebidas houve uma ampliação muito grande do volume de vendas e expansão de mercado com a adoção do programa.

Empresas nas quais os funcionários nunca escondem suas emoções, não existe a presença do individualismo nas relações profissionais, e há liberdade para se questionar as ordens de seus dirigentes são aquelas que tiveram redução considerável de custos a partir da adoção do programa de sustentabilidade.

No setor brasileiro de cosmético verifica-se que a cultura organizacional pode ser entendida como uma fonte de vantagem competitiva, pois as empresas que conseguirem implementar melhores práticas de gestão, com menor nível de rigidez hierárquica, centralização e maior capacidade de absorver novos conhecimentos e inovação tem associação com empresas que tem melhores resultados em termos de sustentabilidade corporativa. Adicionalmente verifica-se ainda que em ambientes com cultura mais fechada os resultados são menos percebidos.

O estudo permitiu identificar elementos relevantes da cultura organizacional existentes no setor de cosmético brasileiro, mas não esgota as possibilidades de análise, que podem ser aprofundadas e comparadas com os resultados de outras abordagens. Como sugestão para futuras pesquisas no setor, fica a possibilidade de aplicação de outros ferramentais estatísticos, e a comparação dos resultados auferidos com aqueles verificados na pesquisa. Há também a expectativa de replicação do survey em outros setores produtivos, a fim de se verificar se os resultados acerca de cultura organizacional são característicos de cada atividade, ou se há um padrão de tendência ou repetição do mesmo ao longo das diversas atividades. 


\section{Referências}

ALCÂNTARA, V. C. et al. Mensuração da Cultura Organizacional: Uma análise quantitativa comparativa. Revista Eletrônica de Ciência Administrativa - RECADM, Campo Largo, v. 8, n. 2, p. 213-232, 2010. Disponível em: <http://revistas.facecla.com.br/index.php/recadm/article/view/621/493 >. Acesso em: 30 nov. 2012.

AZAPAGIC, A. Systems approach to corporate sustainability: a general management framework. Institution of Chemical Engineers, New Jersey, v. 81, n. 5, p. 303-316, 2003.

BANSAL, P. Evolving sustainably: A longitudinal study of corporate sustainable development. Strategic Management Journal, New York, v. 26, n. 3, p. 197-218, 2005.

BANSAL, P.; ROTH, K. Why Companies Go Green: A Model of Ecological Responsiveness. Academy of Management Review, New York, v. 43, n. 4, p. 717-736, aug. 2000.

BAUMGARTNER, R. J. Managing Corporate Sustainability and CSR: A Conceptual Framework Combining Values, Strategies and Instruments Contributing to Sustainable Development. Corporate Social Responsibility and Environmental Management, v. 21, ed. 5, p. 258-271, 2014.

BERNS, M., TOWNEND, A., KHAYAT, Z., BALAGOPAL, B., REEVES, M., HOPKINS, M., \& KRUSCHWITZ, N. The Business of Sustainability. MITSloan Management Review, 2012.

CHIAPPETTA JABBOUR, C. J.; BORGES DE OLIVEIRA, S. V. W,; CASTRO, R. Cultura Organizacional, Inovação e Gestão Ambiental: Integrando Conceitos para a Edificação de Organizações Sustentáveis. v. 3, n. 3, p. 1-10, 2011.

CHIAPPETTA JABBOUR, C. J.; ALMADA SANTOS, F. C.; FONSECA, S. A.; et al. Green teams: understanding their roles in the environmental management of companies located in Brazil. Journal of Cleaner Production. v. 46, p. 58-66, 2013.

CHIAVENATO, I. Gestão de pessoas. 3. ed. Rio de Janeiro: Elsevier, 2010. 579 p.

CRANE, A. Rhetoric and reality in the greening of organizational culture. Greener Management International, Sheffield, v. 11, n. 12, p. 49-62, 1995.

DUBOIS, C. L. Z.; DUBOIS, D. A. Strategic Hrm As Social Design For Environmental Sustainability In Organization Human Resource Management. v. 51, ed. 6, p. 799-826, 2012.

DYLLICK, T.; HOCKERTS, K. Beyond the Business Case for Corporate Sustainability. Business Strategy and the Environment, v. 11, n. 2, p. 130-141, 2002.

ELKINGTON, J. Canibais com garfo e faca. São Paulo: Makron, 2001. p. 421.

ELKINGTON, J. Towards the sustainable corporation: win-win-win business strategies for sustainable development. California Management Review, Berkeley, v. 36, n. 2, p. 90-101, 1997.

EPSTEIN, M. J.; ROY, M-J. Sustainability in action: identifying and measuring the key performance drivers. Long Range Planning, v. 34, n. 5, p. 585-604, 2001.

EPSTEIN, M. J.; WISNER, P. S. Using the balanced scorecard approach to implement sustainability. Environmental Quality Management, v. 11, n. 2, p. 1-10, 2001.

FLEURY, M. T.; FLEURY, A. Aprendizagem e Inovação Organizacional. 2 ed. São Paulo: Atlas, 1997. 


\section{AVALIAÇÃO DA SUSTENTABILIDADE CORPORATIVA E DA CULTURA ORGANIZACIONAL - SURVEY NO SETOR BRASILEIRO DE COSMÉTICOS}

HARRIS, L. C.; CRANE, A. The greening of organizational culture: Management views on the depth, degree and diffusion of change. Journal of Organizational Change Management, v. 15, n. 3, p. 214-234, 2002.

HART, S. L.; MILSTEN, M. B. Criando valor sustentável. Revista de Administração de Empresas - RAE Executivo, São Paulo, v. 3, n. 7, p. 65-79, maio/jun. 2004. Disponível em: < http://www.is.cnpm.embrapa.br/bibliografia/2004 Criando valor sustentavel.pdf>. Acesso em: 20 dez. 2012.

HAIR, J. F. et al. Multivariate Data Analysis. 6. ed. New Jersey: Prentice Hall, 2006. 928 p.

HOFSTEDE, G. Culture's consequences: international differences in work-related values. Beverly Hills: Sage, 1980. 475 p.

HOFSTEDE, G. Culture and organizations. International Studies of Management and Organizations, New York, v. 10, n. 4, p. 15-41, 1981.

HOFSTEDE, G. Identifying organizational subcultures: An empirical approach. Journal of Management Studies, v. 35, n.1; p. 1-12, 1998.

HOMBURG, C.; PFLESSER, C. A multiple-layer model of market-oriented organizational culture: measurement issues and performance outcomes. Journal of Marketing Research, Chicago, v. 37, n. 4, p. 449-462, nov. 2000.

HUMPHREYS, A. How is Sustainability Structured? The Discursive Life of Environmentalism. Journal Of Macromarketing. V. 34, ed. 3, p. 265-281, 2014.

INSTITUTO ETHOS. 0 papel dos institutos e fundações na atuação socialmente responsável da empresa. São Paulo: Instituto Ethos de Empresas e Responsabilidade Social, 2012. Disponível em: $\quad$ http://www.siteuniethos.org.br/wpcontent/uploads/2009/10/Publica\%C3\%A7\%C3\%A3o-Institutos-e-

Funda\%C3\%A7\%C3\%B5es-Uniethos-Vale.pdf/. Acesso em: 27 abr. 2013.

ISAKSSON, R. Total quality management for sustainable development: process based system models. Business Process Management Journal, v. 12, n. 5, p. 632-645, 2006.

JACKSON, S. E., SCHULER, R. S., LEPAK, D., TARIQUE, I. Human resource management practice and scholarship: A North American perspective. In C. Brewster and W. Mayrhofer (eds.), Handbook of Research in Comparative Human Resource Management. 2011.

KAPTEIN, M.; WEMPE, J. The balanced company: a theory of corporate integrity. Oxford: Oxford University Press, 2002.360 p.

KIRON, D.; KRUSCHUWITZ, N.; RUBEL, H.; REEVES, M.; FUISZ-KEHRBACH, S. K.; Sustainability's Next Frontier: Walking talk on the sustainability issues that matter most. MITSloan Management Review in collaboration with The Boston Consulting Group, Boston, 2014.

LINNENLUECKE, M. K.; GRIFFITHS, A. Corporate sustainability and organizational culture. Journal of World Business, v. 45, n. 4, p. 357-366, 2010.

LINNENLUECKE, M. K.; RUSSELL, S. V.; GRIFFITHS, A. Subcultures and sustainability practices: the impact on understanding corporate sustainability. Business Strategy and the Environment, Queensland, v. 18, n. 7, p. 432-452, 2009.

MARTINS, R. A. Abordagens quantitativa e qualitativa. In: MIGUEL, P. A. C. (org.). Metodologia 


\section{André Luiz Romano, Isabela Tatiana Teixeira, Alceu Gomes Alves Filho \\ André Luís Helleno}

de pesquisa em engenharia de produção e gestão de operações. Rio de Janeiro: Elsevier, 2010, p.45-61.

MENDIOLA, I. S.; BELTRAN, A. G.; TIRADOS, R. M. G. Evaluation and implementation of social responsibility. The Service Industries Journal. v. 33, n. 9-10, p. 846-858, 2013.

MOTTA, F. P.; CALDAS, M. P. Cultura organizacional e cultura brasileira. São Paulo: Atlas, 1997. 325 p.

OUCHI, W. G.; PRICE, R. L. Hierarchies, clans, and theory Z: A new perspective on organization development. Organizational Dynamics, v. 21, n. 4, p. 62, 1993.

ROMANO, A. L. Um estudo sobre indicadores de sustentabilidade corporativa: análise de ferramentas e verificação da aplicação numa empresa de material de escritório. $173 \mathrm{f}$.. Dissertação (Mestrado em Desenvolvimento Regional), Centro Universitário de Araraquara. Araraquara, 2010.

ROMAnO, A. L. Proposta de Modelo de Avaliação da Sustentabilidade Corporativa: As Práticas no Setor de Cosmético Brasileiro. $204 \mathrm{f}$. Tese (Doutorado em Engenharia de Produção), Universidade Metodista de Piracicaba. Santa Bárbara d'Oeste, 2014.

SACHS, I. Caminhos para o desenvolvimento sustentável. Rio de Janeiro: Garamond, 2000. 96 p.

SALZMANN, O.; IONESCU-SOMERS, A.; STEGER, U. The Business Case for Corporate Sustainability: European Management Journal, v. 23, n. 1, p. 27-36, fev. 2005.

SAVITZ, A. W.; WEBER, K. A Empresa Sustentável: o verdadeiro sucesso é o lucro com responsabilidade social e ambiental. Rio de Janeiro: Campus/Elsevier, 2007.

SCHEIN, E. H. Organizational Culture and Leadership: a dynamic view. San Francisco: JosseyBass, $1992.380 \mathrm{p}$.

SCHEIN, E. H. Organizational Culture and Leadership. 3. ed. San Francisco: Jossey-Bass, 2004. $448 \mathrm{p}$.

SCHWARTZ, H.; DAVIS, S. M. Matching corporate culture and business strategy. Organizational Dynamics, v. 10, n. 1, p. 30-48, 1981.

SHRIVASTAVA, P. The role of corporations in achieving ecological sustainability. Academy of Management Review, New York, vol. 20, n. 4, p. 936-960, out. 1995.

SILVA, L. M. T.; MEDEIROS, C. A.; ENDERS, W. T. Avaliação da Cultura Organizacional: um contraponto entre as abordagens quantitativas e qualitativas. INTERFACE - Revista do Centro de Ciências Sociais Aplicadas, Natal, v. 8, n. 2, p. 124-140, 2011. Disponível em: <http://ccsa.ufrn.br/ojs/index.php/interface/article/view/463/430>. Acesso em: 05 dez. 2012.

SMIRCICH, L. Concepts of culture organizational analysis. Administrative Science Quarterly, v.28, n.3, p.339-358, sep. 1983.

SRIVASTAVA, M.; FRANKLIN, A.; MARTINETTE, L. Building a Sustainable Competitive Advantage Journal of technology management \& innovation, v. 8, ed. 2, p. 47-60, 2013.

TRICE, H. M., BEYER, J. M. Studying Organizational Cultures Through Rites and Ceremonials. Academy of Management Review, New York, vol. 9, n. 4, p. 653-669, oct.1984.

VEIGA, J. E. A emergência socioambiental. São Paulo: SENAC SP, 2007. 144 p.

WAGNER, M. Corporate social performance and innovation with high social benefits: A 
quantitative analysis. Journal of Business Ethics, New York, v. 94, n. 4, p. 581-594, 2010.

WEINSTEIN, M. P., TURNER, R. E., \& IBANEZ, C. The Global Sustainability Transition: It is more than changing light bulbs. Sustainability: Science, Practice, \& Policy. 2013.

WRIGHT, C.; NYBERG, D. Working with passion: Emotionology, corporate environmentalism and climate change. Human Relations. v. 65, n. 12, p. 1561-1587, 2012.

ZELLWEGER, T. M.; NASON, R. S.; NORDQVIST, M.; et al. Why Do Family Firms Strive for Nonfinancial Goals? An Organizational Identity Perspective. Entrepreneurship Theory And Practice, v. 37, n. 2, p. 229-248, 2013. 